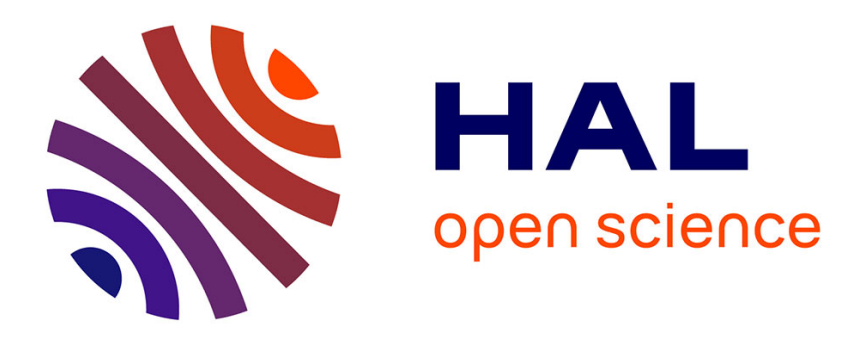

\title{
Supersymmetry results at the Tevatron
}

F. Badaud

\section{To cite this version:}

F. Badaud. Supersymmetry results at the Tevatron. DIS2006 XIV International Workshop on Deep Inelastic Scattering, Apr 2006, Tsukuba, Japan. in2p3-00104934

HAL Id: in2p3-00104934 https://hal.in2p3.fr/in2p3-00104934

Submitted on 9 Oct 2006

HAL is a multi-disciplinary open access archive for the deposit and dissemination of scientific research documents, whether they are published or not. The documents may come from teaching and research institutions in France or abroad, or from public or private research centers.
L'archive ouverte pluridisciplinaire HAL, est destinée au dépôt et à la diffusion de documents scientifiques de niveau recherche, publiés ou non, émanant des établissements d'enseignement et de recherche français ou étrangers, des laboratoires publics ou privés. 


\title{
SUPERSYMMETRY RESULTS AT THE TEVATRON
}

\author{
FRÉDÉRIQUE BADAUD \\ on behalf of the $C D F$ and $D \emptyset$ collaborations \\ Laboratoire de Physique Corpusculaire-IN2P3/CNRS, Clermont-Ferrand, France \\ E-mail: badaud@clermont.in2p3.fr
}

\begin{abstract}
The results for searches for Supersymmetry by the CDF and $\mathrm{D} \emptyset$ collaborations in $\mathrm{p} \overline{\mathrm{p}}$ collisions at $\sqrt{s}=1.96 \mathrm{TeV}$ are presented here. Searches for chargino/neutralino and the lightest stop, as well as scenarios with R-parity violation are focused here. The integrated luminosity analyzed ranges from 300 to $800 \mathrm{pb}^{-1}$ depending on the search. Further informations can be found on the public web pages of the two experiments ${ }^{1}, 2$.
\end{abstract}

\section{Supersymmetry}

Supersymmetry (SUSY) predicts the existence of a new particle for each of the Standard Model (SM) particles, differing by half a unit in spin but otherwise sharing same quantum numbers. A discrete multiplicative symmetry, called R-parity, is defined as $R p=(-1)^{(2 S+3 B+L)}$ where $B$ is the baryon number, $L$ is the lepton number and $S$ is the spin of the particle, such that a SM particle carries $R p=+1$ and a SUSY particle $R p=-1$. Supersymmetric particles have not been observed yet implying that SUSY is a broken symmetry. In $R p$ conserving models, supersymmetric particles are produced in pair and the lightest supersymetric particle (LSP) is stable. The masses for the proposed superpartners are potentially accessible at the Tevatron.

\section{Charginos and neutralinos}

\subsection{Multilepton final state}

In the mSUGRA scenario the superparticles are produced in pairs and the lighter charginos and neutralinos, mixed state of electroweak gauginos and higgsinos, and the sleptons, are less massive than gluinos and squarks. The LSP is the $\tilde{\chi}_{1}^{0}$. In the case where charginos and neutralinos decay leptonically, $\tilde{\chi}_{1}^{ \pm} \rightarrow \ell^{ \pm} \nu_{\ell} \tilde{\chi}_{1}^{0}$ and $\tilde{\chi}_{2}^{0} \rightarrow \ell^{ \pm} \ell^{\mp} \tilde{\chi}_{1}^{0}$, very clean final states of 
three leptons plus missing energy $\left(\mathrm{E}_{\mathrm{T}}\right)$ caused by the $\tilde{\chi}_{1}^{0}$ 's and the neutrino are expected. Very few SM processes contribute to such a signature, the dominant being Drell-Yan plus a misidentified jet or a $\gamma \rightarrow e^{+} e^{-}$conversion, and real WZ production. New CDF results on trileptons are summarized in Table 1. No significant excess is observed. A previous $\mathrm{D} \emptyset$ analysis sets a limit of $117 \mathrm{GeV} / \mathrm{c}^{2}$ on the $\tilde{\chi}_{1}^{ \pm}$mass considering a mSUGRA scenario with sleptons slightly heavier than the $\tilde{\chi}_{1}^{ \pm}$and no sleptons mixing.

Table 1. CDF results of search for $\tilde{\chi}_{1}^{ \pm} \tilde{\chi}_{2}^{0} \rightarrow \ell^{ \pm} \ell^{\mp}+X$. LS means Like Sign.

\begin{tabular}{cccccc}
\hline Channel & $\mu \mu+\ell$ & $\mu e+\ell$ & LS ee & LS $\mu \mu$ & LS $\mu e$ \\
Luminosity & $745 \mathrm{pb}^{-1}$ & $700 \mathrm{pb}^{-1}$ & $704 \mathrm{pb}^{-1}$ & $704 \mathrm{pb}^{-1}$ & $704 \mathrm{pb}^{-1}$ \\
\hline $\begin{array}{c}\text { SM expectation } \\
\text { observed }\end{array}$ & $0.64 \pm 0.18$ & $0.78 \pm 0.11$ & $2.6 \pm 0.4$ & $3.5 \pm 0.6$ & $0.7 \pm 0.1$ \\
& 1 & 0 & 4 & 5 & 0 \\
\hline
\end{tabular}

\subsection{Diphotons}

In another SUSY model, Gauge Mediated Supersymmetry Breaking (GMSB), the LSP is the gravitino, the super partner of the graviton. Each of the $\tilde{\chi}_{1}^{0}$ 's from chargino-neutralino production will decay into a gravitino and a photon. The signal is a final state with two energetic photons and large $\mathrm{E}_{\mathrm{T}}$. The physical backgrounds are estimated negligible. Instrumental backgrounds come from QCD processes with either real $\gamma$ or jets misidentified as $\gamma$ and $W(\rightarrow e \nu) \gamma$ and $W(\rightarrow e \nu) j e t$ production where the electron and the jet are misidentified as a photon. $\mathrm{D} \emptyset$ has searched for this signature in $760 \mathrm{pb}^{-1}$ of data. 2 events are observed with an expected background of $2.1 \pm 0.7$ events. An upper limit, see Figure 1, is set on GMSB SUSY production.

\section{Squarks and gluinos}

\subsection{Stops}

In many scenarios, the lightest stop quark, $\tilde{t}_{1}$, is expected to be the lightest of all the squarks. Due to their strong couplings, squarks and gluinos are expected to be produced abundantly at hadron colliders. $\mathrm{D} \emptyset$ has looked for final states $e^{ \pm} \mu^{\mp}+b \bar{b}+\mathrm{E}_{\mathrm{T}}$ and $\mu^{ \pm} \mu^{\mp}+b \bar{b}+\mathrm{E}_{\mathrm{T}}$, which is the signature of $\tilde{t}_{1}$ pair production, followed by $\tilde{t}_{1} \rightarrow b \ell \tilde{\nu}$, which is expected to be the dominant decay mode when $m_{\tilde{\nu}} \approx m_{W}$. For the $e \mu$ final state, two regions of $\Delta M=m_{\tilde{t}_{1}}-m_{\tilde{\nu}}$ are examined. The expected number of total background 
is $23.0 \pm 3.1(40.7 \pm 4.4)$ for $21(42)$ observed in the $e \mu$ low $\Delta m$ (high $\Delta m$ ) region. In the $\mu \mu$ final state the expectation from SM is $2.88 \pm 0.43_{-0.04}^{+0.10}$ while 1 event is observed. All the results are compatible with SM expectations. The regions for which the calculated cross section upper limit is smaller than the theoritical cross section are $95 \%$ C.L. excluded and are shown in Figure 2.

\section{R-parity violation}

CDF looked for $R p$ violating SUSY in the multilepton channel. The search is performed under the assumption that SUSY particles are pair produced and decay under $R p$ conservation while only the LSP can decay into two charged leptons plus a neutrino via the $\lambda_{121}$ or $\lambda_{122}$ coupling. Both the 3 and 4 leptons signatures, $e e \ell(\ell)$ and $\mu \mu \ell(\ell)$ with $\ell=e$ or $\mu$, were investigated and the observation is compatible with the expectation. As an example, Figure 4 shows the invariant mass distribution of the leading leptons. The channels are combined and upper limits at 95\% C.L. on the cross sections, $\sigma<0.21 \mathrm{pb}$ for $\lambda_{121}>0$ and $\sigma<0.11 \mathrm{pb}$ for $\lambda_{122}>0$, are obtained.

\subsection{Long-lived LSP}

$\mathrm{D} \emptyset$ looked for the decay of the neutralino to leptons and a neutrino in 383 $\mathrm{pb}^{-1}$ of data. The analysis focuses on the scenario where the $R p$ coupling is weak and the LSP would travel $\geq 5 \mathrm{~cm}$ before decaying. This possibility was inspired by an excess in dimuon events reported by NuteV. No events are observed with an expectation of $0.8 \pm 1.1 \pm 1.1$ from backgrounds and the limit set excludes the possibility that the NuteV events are due to neutralino decay.

\section{Conclusions}

CDF and D $\emptyset$ have searched for SUSY and no deviation from the Standard Model have been found. SUSY parameter regions have been excluded. High luminosity samples, corresponding to $1 \mathrm{fb}^{-1}$ are being analyzed.

\section{References}

1. http://www-cdf.fnal.gov/physics/exotic/exotic.html

2. http://www-d0.fnal.gov/Run2Physics/WWW/results/np.htm 


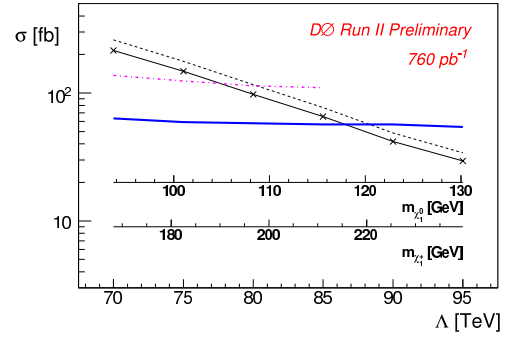

Figure 1. 95\% C.L. limit on GMSB SUSY Snowmass Slope obtained in the analysis (thick blue line) and in the previous $\mathrm{D} \emptyset$ result (dot-dashed) purple-line. SUSY LO (NLO) cross-section is shown in black solid (dashed) line.

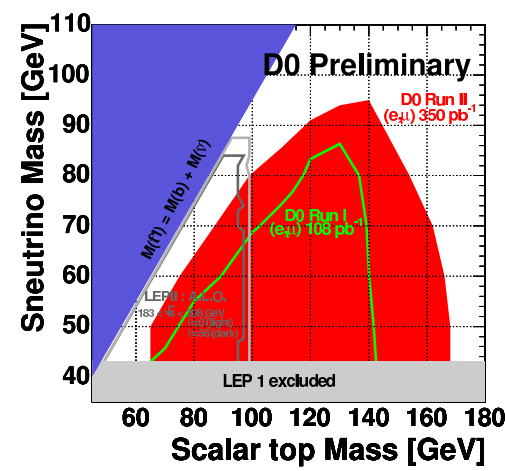

Figure 2. $95 \%$ C.L. excluded region in the stop search for the combination of $e \mu$ and $\mu \mu$ final state.

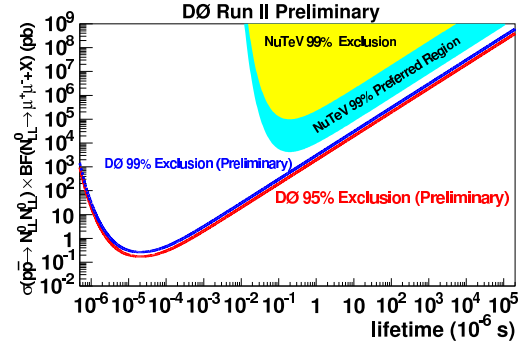

Figure 3. The red curve is the $95 \%$ C.L. limit on pair production of neutral, longlived particles.

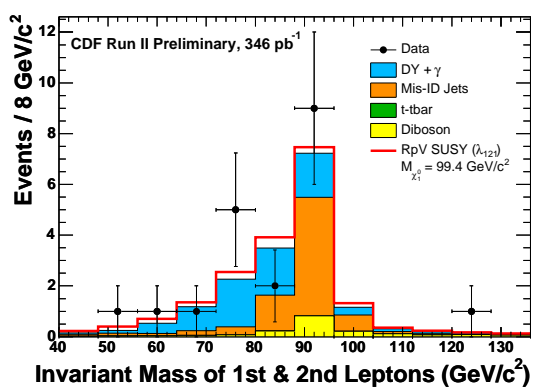

Figure 4. Search for $R p$ violating SUSY in the multilepton channel. Invariant mass distribution of the leading leptons. 